\title{
Statin-Associated Muscle Disease: Advances in Diagnosis and Management
}

\author{
Beth A. Taylor ${ }^{1,2,3}$ (D) Paul D. Thompson ${ }^{1,3}$ \\ Published online: 24 September 2018 \\ (C) The American Society for Experimental NeuroTherapeutics, Inc. 2018
}

\begin{abstract}
Since the first approval of lovastatin in 1987, hydroxy-methyl-glutaryl CoA (HMG CoA) reductase inhibitors, or statins, have been effective and widely popular cholesterol-lowering agents with substantial benefits for the prevention and treatment of cardiovascular disease. Not all patients can tolerate these drugs, however, and statin intolerance is most frequently associated with a range of side effects directed toward skeletal muscle, termed statin-associated muscle symptoms or SAMS. SAMS are particularly difficult to treat because there are no validated biomarkers or tests that can be used to confirm patient self-reports of SAMS, and a number of patients who report SAMS have non-specific muscle pain not attributable to statin therapy. This review summarizes the most recent evidence related to diagnosis and management of SAMS. First, the range of skeletal muscle side effects associated with statin therapy is described. Second, data regarding the incidence and prevalence of SAMS, the most frequently experienced muscle side effect, are presented. Third, the most promising new techniques to confirm diagnosis of SAMS are explored. Finally, the most effective strategies for the clinical management of SAMS are summarized. Better diagnostic and treatment strategies for SAMS will increase the number of patients using these life-saving statins, thereby increasing statin adherence and reducing the costs of avoidable cardiovascular events.
\end{abstract}

Key Words Statins $\cdot$ Statin-associated muscle symptoms $\cdot$ Skeletal muscle $\cdot$ Myalgia $\cdot$ Statin intolerance

\section{Introduction}

Hyperlipidemia is a major public health problem. Approximately $30 \%$ of US adults have elevated low-density lipoprotein cholesterol (LDL-C), which doubles their heart disease risk [1]. Hydroxy-methyl-glutaryl (HMG) coenzyme A reductase inhibitors or statins are effective in reducing LDL-C and decrease the incidence of cardiac events by 20 to $44 \%$ for both secondary [2] and primary prevention [3]. The release of the 2013 American College of Cardiology and the American Heart Association (ACC-AHA) guidelines of the treatment of cholesterol expanded the number of US

Beth A. Taylor

Beth.Taylor@uconn.edu

1 Division of Cardiology, Hartford Healthcare, Hartford, CT, USA

2 Department of Kinesiology, University of Connecticut, Storrs, CT, USA

3 University of Connecticut School of Medicine, Farmington, CT, USA adults eligible for statin therapy from 43.2 million (37.5\% of US adults) to 56.0 million (48.6\%) [4]. According to the Centers for Disease Control, $26 \%$ of US adults $>40$ years of age and $48 \%$ of adults $>75$ years of age report using a cholesterol-lowering drug, with $93 \%$ of respondents using a statin [1]. In addition, it has been estimated that $49.7 \%$ of US adults at high CVD risk ( $\geq 20 \% 10$ year CVD risk) are not receiving statins [5]. Collectively, these data support an indication for continued widespread, and likely increasing, statin use.

Statins are well-tolerated in most adults, with few common serious adverse effects. However, they are associated with a range of skeletal muscle side effects, ranging from mild to severe. Among the serious but rare muscle side effects are rhabdomyolysis and statin-induced necrotizing autoimmune myopathy (SINAM). Rhabdomyolysis is typically diagnosed as a creatine kinase (CK) level $>10$ times the upper limit of normal, with evidence of renal compromise, and without other causes of muscle injury. The incidence of statin-associated rhabdomyolysis is estimated to be 2.0 cases per 10,000 person-years of treatment and ranged from 0.3 cases for lovastatin to 8.4 cases for cerivastatin $[6,7]$. Cerivastatin has been 
removed from the market because of its rhabdomyolysis risk so that the current incidence of rhabdomyolysis is $\sim 1$ case per 10,000 person-years. The rhabdomyolysis generally associated with statin use resolves with cessation of the statin. By contrast, SINAM is an autoimmune myopathy classified by the presence of a myositis-specific autoantibody against 3hydroxy-3-methylglutaryl-coenzyme A reductase (HMGCR) [8]. SINAM is distinct from, but in rare cases can cause, statinassociated rhabdomyolysis. SINAM patients present with proximal muscle weakness, markedly elevated CK levels, and persistence (or often worsening) of symptoms and CK elevations despite drug discontinuation. Muscle biopsies show myonecrosis often with few inflammatory cells [9]. Antibodies against HMGCR are detected in 94\% of patients with SINAM [10] and distinguish it from other related idiopathic inflammatory myopathies, although it should be noted that a number of anti-HMGCR myopathy patients do not have a history of exposure to statin drugs [8]. These statinunexposed adult patients are generally younger and tend to have more severe disease and worse prognosis than the classic statin-exposed cohort [11]. Regardless, anti-HMGCR autoantibodies are highly specific and are not found in patients with other muscle diseases (such as self-limited statin intolerance or muscular dystrophy), although they can co-occur with other myositis-specific autoantibodies in patients with multiple autoimmune disorders [8]. Immunosuppressive therapy is required to prevent progression to severe, often irreversible, muscle weakness and wasting in patients with SINAM. SINAM is estimated to occur in 2-3 of 100,000 statin users, although HMGCR antibodies are not routinely measured and the clinical presentation of rhabdomyolysis makes estimation of incidence difficult [10].

Statins are most frequently associated with mild muscle complaints, termed statin-associated muscle symptoms or SAMS, which include myalgia, cramps, and perceived weakness. These symptoms are the most difficult to diagnose because the time from statin initiation to symptoms, the type of complaints, and their severity varies dramatically among patients, and there is no confirmatory diagnostic test for SAMS. Moreover, a large number of patient self-reports of SAMS appear to be due to non-specific muscle pain, and/or the socalled nocebo or expectation of harm effect. Treatment strategies are largely based on either drug cessation or alteration in the type or dose of statin until the patient reports that symptoms are absent, diminished, or tolerable. Despite these treatment strategies, $62 \%$ of former statin users reported stopping statin therapy because of side effects [12], making SAMS a serious public health problem. Patients stopping [13] or altering their statin use [14] due to intolerance have a markedly increased risk of cardiovascular events. Patients who are statin intolerant and/or less adherent to statin therapy incur greater healthcare costs because of their higher rate of cardiovascular events $[15,16]$. Indeed, it has been estimated that expanding statin use in the USA to the 5.27 million untreated high-risk and 20.29 million untreated moderate-risk adults would prevent 384,000 and 616,000 CVD events, respectively, over 10 years [5].

The present review focuses on updates in the diagnosis and treatment of SAMS and addresses the 3 critical questions on how to better manage SAMS in clinical practice.

\section{What Is the True Rate of SAMS?}

Estimates of SAMS have varied from $<1$ to $>20 \%$ of patients, in part because many of the initial reports of SAMS originated from observational studies, or clinical case compilations from lipid specialists. For example, the PRedIction of Musular Risk in Observational Conditions or PRIMO study obtained questionnaires on 7924 French patients treated for at least 3 months with fluvastatin $80 \mathrm{mg}$, atorvastatin $40-80 \mathrm{mg}$, pravastatin $40 \mathrm{mg}$, or simvastatin 40-80 mg daily [17]. Muscular symptoms were reported by $10.5 \%$ of participants, but this was an observational, unblinded, uncontrolled retrospective study. Similarly, a retrospective analysis of National Health and Nutrition Examination Survey data reported that $22 \%$ of respondents with a statin prescription reported muscle pain, while $16.7 \%$ of non-statin users reported muscle pain [18].

Randomized controlled, double-blinded clinical trials of statin therapy have also failed to establish a rigorous SAMS prevalence in participants, possibly because of study design. A systematic review identified 1012 reports of statin randomized controlled clinical trials [19]. Among 42 trials which qualified for detailed analysis, only 4 reported average CKs, and only 1 queried participants specifically for muscle symptoms using predefined criteria. A total of 26 studies reported muscle symptoms, which occurred in $12.7 \%$ and $12.4 \%$ of statin- and placebo-treated subjects, respectively. This small difference approached statistical significance $(p=0.06)$ due to the large sample size.

Even in the few studies designed to rigorously assess SAMS, true prevalence rates are confounded by the fact that a substantial portion of SAMS are probably due to nonspecific muscle pain; that is, pain not directly attributable to the effects of statins on skeletal muscle. For example, in our The Effect of Statins on Muscle Performance, or STOMP study, we randomized 420 statin-naïve subjects to either placebo or atorvastatin $80 \mathrm{mg}$ daily for 6 months. STOMP used a predefined definition of myalgia which required subjects to report unexplained new or increased myalgia, cramps, or muscle aching that lasted at least 2 weeks, resolved within 2 weeks of treatment cessation, and returned within 4 weeks of drug reinitiation. Subjects were called every 2 weeks and queried about muscle symptoms. Twenty-three atorvastatin and 14 placebo subjects reported new, unexplained muscle pain $\left(\chi^{2}=3.16, p=0.08\right)$. Of these, 19 atorvastatin and 10 placebo 
subjects met the study myalgia definition $\left(\chi^{2}=3.74 ; P=\right.$ 0.054 ) [20]. This suggests that only approximately half of the subjects whose pain is attributable to statins actually have statin-induced symptoms.

In our more recent Coenzyme Q10 in Statin Myopathy study, we used a double-blind, placebo-controlled, crossover protocol to confirm SAMS in 120 patients with a history of statin-associated muscle complaints [21]. After a 4-week washout period from all cholesterol-lowering drugs, the subjects were randomized to simvastatin $20 \mathrm{mg}$ daily or placebo for 8 weeks, washed out for 4 weeks, and then crossed over to alternative therapy. Only $35.8 \%$ of patients $(n=43)$ experienced myalgia on simvastatin and did not experience myalgia on placebo, what was termed true or confirmed statin myalgia, and $17.5 \%$ of patients $(n=21)$ had no symptoms on simvastatin or placebo. However, 29.2\% $(n=35)$ experienced pain on placebo but not on simvastatin and $17.5 \%(n=21)$ experienced pain on both simvastatin and placebo during the confirmation phase. In the similarly designed Goal Achievement After Utilizing an Anti-PCSK9 Antibody in Statin-Intolerant Subjects 3 (GAUSS-3) clinical trial, patients with a history of intolerance to 2 or more statins were washed out for 4 weeks, then randomized to either atorvastatin $20 \mathrm{mg}$ or placebo for 10 weeks. A 2-week washout period separated the cross-over to the alternative treatment. In GAUSS-3, 209 of 472 patients who completed both phases of the run-in (42.6\%) with a history of muscle-related side effects reported SAMS on $20 \mathrm{mg}$ atorvastatin alone whereas 130 of the 491 patients (26.5\%) reported SAMS on placebo alone. An additional $48 \%$ or $9.8 \%$ of patients had symptoms on both treatments, and $85 \%$ or $17.3 \%$ of patients experienced no muscle pain on either treatment [22]. Collectively, then, data from STOMP, CoQ10, and GAUSS-3 support that there is a high percentage of false positive (or non-specific) muscle complaints associated with SAMS, suggesting that 30 to $50 \%$ of patients who report SAMS may have another source of muscle pain besides their statin drug.

These reports over the entire range of clinical and observational trials are so discrepant and unreliable that some researchers and clinicians have stated that SAMS do not exist at all and instead are solely a product of the "nocebo effect" [23], citing recent clinical trial data from the AngloScandinavian Cardiac Outcomes Trial-Lipid-Lowering Arm (ASCOT-LLA) showing similar rates in SAMS during double-blind $10 \mathrm{mg}$ /day atorvastatin versus placebo administration $(2.03 \%$ vs $2.00 \%)$. These rates only differed when patients shifted from double-blind to open-label statin administration; at this point, patients on statin therapy experienced a higher rate of SAMS than patients not taking them. Specifically, muscle-related AEs were reported at a significantly higher rate by participants taking statins than by those who were not (1.26\% vs $1.00 \%$ ) [24]. However, a closer look at this trial yields an alternative interpretation of the data. If
SAMS were solely attributable to a nocebo effect, then it would be expected that the rate of SAMS in patients taking atorvastatin would increase following the shift from blinded administration to open-label administration. Instead, the rate of SAMS decreased (from 2.03 to $1.26 \%$ ), and thus these data are not wholly convincing of the premise that SAMS are attributable only to a nocebo effect. Moreover, combined numbers and percentages of SAMS classifications from the CoQ10 and GAUSS-3 trials as described above are shown in Table 1 . As $42.6 \%$ of patients with a previous history of SAMS experience confirmed SAMS on statin alone and $27.9 \%$ experience SAMS on placebo alone, it might be inferred that the $\sim 15 \%$ difference between these 2 groups indicates that SAMS are due to more than a nocebo effect.

Of critical importance, then, for better addressing this question are trials aimed at elucidating SAMS rates and distinguishing between confounding factors such as nonspecific muscle pain, the nocebo effect, statin-drug interactions that evoke muscle pain, and underlying myopathies. To this end, a newly designed statin web-based investigation of side effects (StatinWISE) is promising, as the study comprises a series of randomized controlled N-of-1 trials comparing atorvastatin and placebo in the primary care setting [25]. Two hundred patients who have discontinued or are considering discontinuing statin therapy due to SAMS will be recruited and exposed to 62 -month treatment periods during which they will receive atorvastatin $20 \mathrm{mg}$ per day or matched placebo. At the end of each treatment period, patients will rate their muscle symptoms. Although a criticism of this trial is that patients are only exposed to 1 type and dose of statin, which therefore may not reproduce previous SAMS, it establishes a study design that is likely to have utility for better establishing SAMS rates by using patients as their own control.

\section{What Are the Most Effective Methods for Confirming a Diagnosis of SAMS?}

The absence of definitive diagnostic tests requires that the diagnosis of statin myalgia and other mild SAMS be based on clinical criteria [26, 27]. Current criteria support that muscle pain and aching (myalgia), cramps, and weakness are among the most common manifestations of SAMS. For example, in the clinical trial GAUSS-2, $80 \%$ of patients exhibiting statin intolerance reported myalgia, and 39\% described muscle weakness [28]. Symptoms are usually bilateral and involve large muscle groups including the thigh, buttock, back, and shoulder girdle musculature. In contrast, cramping is usually unilateral and may involve small muscles of the hands and feet. Symptoms often appear early after starting stain therapy or after an increase in dose. For example, the median time to onset of symptoms is $\sim 1$ month [29], and in 
Table 1 Comparison of SAMS rates by number and (\%) in CoQ10 and GAUSS-3 trials

\begin{tabular}{llll}
\hline & CoQ10 & GAUSS-3 & Combined \\
\hline SAMS on statin & $43(35.8)$ & $209(44.3)$ & $252(42.6)$ \\
SAMS on placebo & $35(29.2)$ & $130(27.5)$ & $165(27.9)$ \\
No SAMS & $21(17.5)$ & $85(18.0)$ & $106(17.9)$ \\
SAMS on both & $21(17.5)$ & $48(10.2)$ & $69(11.7)$ \\
Total & 120 & 472 & 592
\end{tabular}

SAMS = statin-associated muscle symptoms; CoQ10 = Coenzyme Q10 in Statin Myopathy [21] trial; GAUSS-3 = Goal Achievement After Utilizing an Anti-PCSK9 Antibody in Statin Intolerant Subjects 3 trial [22]; Combined $=$ additive data from both trials

both our STOMP and CoQ10 studies, patients who had confirmed SAMS (i.e., muscle symptoms on statin only) experienced their symptoms in approximately half the time as patients experiencing non-specific or unconfirmed SAMS [20, 21]. In addition, muscle symptoms typically resolve or start to dissipate within 2-4 weeks after cessation of therapy, although it may take several months for symptoms to totally resolve. Persistence of symptoms for more than 2 months after drug cessation suggests an alternative diagnoses or an underlying muscle disease possibly provoked by statin therapy. Different statins usually produce similar symptoms. Some patients do tolerate 1 statin better than another, likely attributable to variations in pharmacodynamics, pharmacokinetics, and pharmacogenomics, although there is currently no clinical test or algorithm to predict a patient's tolerance to different statins [30]. The reappearance of symptoms with statin rechallenge and their disappearance with drug cessation offers the best evidence that the symptoms are truly SAMS [27]. Unfortunately, such clinical drug challenges are unblinded and subjective and especially difficult for the group of patients experiencing the nocebo effect or non-specific muscle pain because reemergence of symptoms does not definitively confirm SAMS.

Few noninvasive assessments exist to accurately quantify these clinical observations, as summarized in Table 2. The most commonly used biomarker is CK, a marker of muscle damage, but SAMS can occur in the absence of clinically elevated CK. Moreover, CK elevations can occur in the absence of SAMS, attributable instead to factors such as medications and recent exercise, and CK itself varies according to age, gender, and ethnicity [26, 31]. Nonetheless, small elevations in CK do occur with statin therapy, and thus serial measurements on a patient could be useful to confirm muscle symptoms, despite often widespread advice against the

Table 2 Potential noninvasive diagnostic tools for SAMS

\begin{tabular}{|c|c|c|}
\hline Diagnostic tool & Description & Evaluation of utility \\
\hline $\begin{array}{l}\text { Diagnosis based on } \\
\text { clinical criteria } \\
\text { alone }\end{array}$ & $\begin{array}{l}\text { Uses patient self-reported description of symptoms (timing of } \\
\text { onset, location of symptoms, time to resolution) to define } \\
\text { likelihood of SAMS based on physician opinion }\end{array}$ & $\begin{array}{l}\text { Requires minimal time and patient burden but is biased by } \\
\text { non-specific muscle pain and the nocebo effect. }\end{array}$ \\
\hline $\begin{array}{l}\text { Statin } \\
\text { challenge-- } \\
\text { dechallenge } \\
\text { protocol }\end{array}$ & $\begin{array}{l}\text { Requires patient to confirm self-reported symptoms by ceasing } \\
\text { statin therapy until symptom resolution and restarting to } \\
\text { test for replication }\end{array}$ & $\begin{array}{l}\text { Unblinded protocol introduces source of bias and patients } \\
\text { with intolerable muscle pain may be unwilling to } \\
\text { rechallenge with the statin. }\end{array}$ \\
\hline Creatine kinase (CK) & $\begin{array}{l}\text { Serologic measurement that must include at least } 1 \text { off-statin } \\
\text { measurement and } 1 \text { on-statin measurement }\end{array}$ & $\begin{array}{l}\text { Small changes may differentiate SAMS but CK measurements } \\
\text { have large variability. }\end{array}$ \\
\hline $\begin{array}{l}\mathrm{CK}+\text { downhill } \\
\text { walking }\end{array}$ & $\begin{array}{l}\text { Serial measurements of CK performed following downhill } \\
\text { walking (eccentric) exercise }\end{array}$ & $\begin{array}{l}\text { May amplify statin-associated changes in CK but protocol } \\
\text { requires equipment, time, and substantial patient burden. }\end{array}$ \\
\hline $\begin{array}{l}\text { Exercise testing } \\
\text { (muscle and/or } \\
\text { aerobic) }\end{array}$ & $\begin{array}{l}\text { Measurements of muscle strength and/or aerobic performance } \\
\text { parameters (respiratory exchange ratio, lactate threshold, } \\
\text { exercise capacity, or maximal oxygen uptake) that must } \\
\text { include at least } 1 \text { off-statin measurement and } 1 \text { on-statin } \\
\text { measurement }\end{array}$ & $\begin{array}{l}\text { Previous studies have not shown measurable differences in } \\
\text { patients with SAMS and protocols require equipment, time, } \\
\text { and substantial patient burden and physical capacity. }\end{array}$ \\
\hline $\begin{array}{l}\text { SAMS-CI } \\
\text { questionnaire }\end{array}$ & $\begin{array}{l}\text { Physician-administered questionnaire that scores SAMS as } \\
\text { probable (score 9-11), possible (score 7-8), and unlikely } \\
\text { (score 2-6) based on } 4 \text { scales of muscle symptom } \\
\text { characteristics: location, pattern, timing of onset, and timing } \\
\text { of improvement after statin withdrawal }\end{array}$ & $\begin{array}{l}\text { Appears useful for identifying patients with non-statin-related } \\
\text { muscle pain (i.e., high negative predictive value) but has not } \\
\text { yet been validated prospectively in a patient population. }\end{array}$ \\
\hline $\begin{array}{l}\text { ACC statin intolerance } \\
\text { tool }\end{array}$ & $\begin{array}{l}\text { Categorizes muscle symptoms in } 8 \text { different categories into a } \\
\text { dichotomous outcome (possible } v s \text { unlikely) for statin } \\
\text { intolerance }\end{array}$ & $\begin{array}{l}\text { Encourages clinician/patient discussion to decide the } \\
\text { likelihood of statin intolerance and the next potential } \\
\text { treatment steps but lacks a scoring system. }\end{array}$ \\
\hline $\begin{array}{l}\text { Indirect measurements } \\
\text { of mitochondrial } \\
\text { function }\end{array}$ & $\begin{array}{l}\text { 31P magnetic resonance spectroscopy and oxygen uptake } \\
\text { kinetics during exercise }\end{array}$ & $\begin{array}{l}\text { Evidence for utility in diagnosing SAMS is lacking to date and } \\
\text { time requirement, equipment cost, and patient burden } \\
\text { associated with testing are substantial. }\end{array}$ \\
\hline
\end{tabular}

Text in italics indicates the most common diagnostic tools currently used. SAMS-CI = Statin-Associated Muscle Symptoms Clinical Index; ACC = American College of Cardiology 
practice. For example, in the STOMP study, patients treated with $80 \mathrm{mg}$ atorvastatin for 6 months exhibited on average a 20-U/L increase in CK that was not observed with placebo [20]. In fact, of the 36 subjects who doubled their CK value at 6 months compared to baseline, twice as many were in the atorvastatin $(n=24)$ than in the placebo $(n=12)$ groups [32]. Moreover, in the Coenzyme Q10 in Statin Myopathy study [21], patients with verified SAMS (i.e., muscle pain on simvastatin only) also exhibited an $\sim 20-\mathrm{U} / \mathrm{L}$ increase in CK that was not observed in patients with pain on placebo only or pain on both placebo and simvastatin [33]. However, most clinical trials have not reported CK on and off treatment except to report as a safety marker the number of patients experiencing CK $>10$ times upper normal limits, so the utility of pre- and post-treatment resting CK is not clear for widescale and individualized diagnostic purposes.

Muscle symptoms may be more frequent in physically active individuals [17], and highly physically active individuals appear less able to tolerate statin therapy [34]. Consequently, because exercise elevates CK, it is possible that a bout of exercise may augment the small CK elevations associated with statin therapy so as to better diagnose SAMS. For example, our previous studies in asymptomatic individuals on statins have demonstrated that 145 -min session of downhill walking at a $-15 \%$ grade evokes a $>200-\mathrm{U} / \mathrm{L}$ greater increase in CK in statin- versus placebo-treated subjects over the following 24-48 h [35, 36]. Several (but not all) studies have also suggested that exercise-associated elevations in CK are greater in asymptomatic patients on statins than those not on statins $[37,38]$, but to our knowledge this model has not been used to diagnose patients with SAMS. Because creatine kinase elevations with exercise are dependent on several mediators such as previous bouts of exercise (which provide a protective effect against future muscle damage) and chronic exposure to physical activity, further research is necessary to determine whether exercise-associated elevations in CK are greater in patients with SAMS and whether there is clinical utility in the exercise model.

Muscle strength testing is an established technique for detecting muscle weakness, and initial reports in patients with and older asymptomatic adults on statins suggested decrements in muscle strength of 10 to $40 \%$ [39, 40]. However, we have used the established, standardized techniques of isokinetic dynamomettry and have not observed changes in muscle strength in healthy adults on high-dose statin therapy [20] and in patients with confirmed SAMS [21], nor have we observed decrements in strength in patients with self-reported SAMS in clinical practice [41]. In addition, muscle strength equipment is expensive and not readily accessible to clinicians and therefore not likely to be widely used in clinical practice to access muscle function in patients with SAMS. Aerobic performance parameters such as maximal oxygen uptake and the ratio of $\mathrm{CO}_{2}$ production to $\mathrm{O}_{2}$ consumption (the "respiratory exchange ratio") also require specialized equipment and personnel and have shown no differences in between patients with and patients without SAMS [20,39].

Mitochondrial dysfunction has been suggested as a mechanism causing SAMS, and multiple studies support this hypothesis [42-45]. For example, atorvastatin treatment evokes the generation of reactive oxygen species (ROS), resulting in a $39 \%$ decrease in maximal mitochondrial respiration in rats [44]. Subsequently, the same researchers then reported greater ROS and mitochondrial apoptosis in glycolytic muscles of patients with SAMS [45]. We also observed that transcriptional patterns between statin myopathic and statin-tolerant subjects differ after eccentric leg exercise; symptomatic subjects treated with a statin exhibited decreased skeletal muscle gene expression for oxidative phosphorylation-related and mitochondrial ribosomal protein genes relative to asymptomatic subjects [46]. Consequently, muscle measurements assessing mitochondrial function may be useful in diagnosing SAMS, although to date a direct relationship between mitochondrial decrements and SAMS severity/symptomology has not been established. Nonetheless, 31P magnetic resonance spectroscopy can be used to assess phosphocreatine recovery kinetics following calf flexion exercise; a slower time course of recovery indicates impaired mitochondrial oxidative function. $\mathrm{Wu}$ et al. found such an impairment in 10 patients treated with statins for 4 weeks, but this study needs to be replicated in a larger clinical trial which is unlikely given the cost and technical expertise necessary to conduct magnetic resonance imaging [47]. Alternatively, the kinetics of pulmonary oxygen uptake during the transition from rest to exercise represent the instantaneous mitochondrial capacity to respond to the metabolic requirements of acute exercise. Slower kinetics, indicative of impaired skeletal muscle oxidative metabolism and mitochondrial function, have been observed in patients with major metabolic diseases [48], but this methodology has not been tested in patients with SAMS and again is costly, difficult, and time-consuming to use clinically.

Systematic deficits in muscle function with SAMS may manifest a blunted response to exercise training. A recent study randomized sedentary, statin-naïve adults to 12 weeks of supervised, aerobic exercise training only or in combination with simvastatin $40 \mathrm{mg}$ daily. Key indicators of the mitochondrial adaptation to endurance exercise including increases in maximal oxygen uptake, citrate synthase activity, and skeletal muscle mitochondrial complexes improved as expected with aerobic exercise training alone but did not improve with exercise training in the simvastatin-treated subjects [49]. However, an aerobic exercise training study is unlikely to be clinically useful to detect SAMS, nor are the measurements of performance (e.g., time to fatigue, maximum power output) routinely used in highly active individuals [50]. So, to date, noninvasive assessments of muscle function have not been successful for evaluating the presence of SAMS and distinguishing 
between real and non-specific statin-associated muscle symptoms.

Of note is that several recent publications have begun to address the diagnostic limitation in distinguishing between SAMS and non-specific muscle symptoms. In 2014, the National Lipid Association Statin Muscle Safety Task Force published a proposed Statin Myalgia Clinical Index (SMCI) that attempted to classify the likelihood of true SAMS [26]. The National Lipid Association expert panel used both research, such as our STOMP trial [20], and clinical experience to create a clinical profile of true statin myalgia. For example, atorvastatin-treated subjects in STOMP with myalgia predominantly reported aching, cramps, or fatigue in the thigh and calf muscles whereas placebo-treated subjects reported generalized fatigue, pain in areas of prior injury, or groin pain. Time from drug initiation to pain onset was half as long in the STOMP atorvastatin-treated subjects as the placebo-treated subjects $(35 \pm 31$ vs $61 \pm 33$ days, $p=0.045)$ so the SMCI assigns more points toward the symptom being true statin myalgia when pain onset $<4$ weeks rather than later onset. The SMCI was updated and revised as the Statin-Associated Muscle Symptoms-Clinical Index (SAMS-CI; Fig. 1) based on author interviews and physician feedback [51]. The SAMS-CI scores SAMS as probable (score 9-11), possible (score 7-8), and unlikely (score 2-6) based on 4 scales of muscle symptom characteristics: location, pattern, timing of onset, and timing of improvement after statin withdrawal. In 2016, a Canadian Working Group proposed several modifications to the original SMCI, based on addition of several more decisive factors such as the existence of muscle pain with nonstatin cholesterol-lowering drugs [31], but to date these proposed modifications have not been incorporated into a published questionnaire. A recent publication validating the SAMS-CI from the Coenzyme Q10 in Statin Myopathy trial [21] reported that patients with confirmed SAMS did indeed exhibit higher scores than patients with muscle symptoms on placebo alone, muscle symptoms on both treatments, or no muscle symptoms on either treatment [52]. While only half of the patients with confirmed SAMS were appropriately classified by the SAMS-CI as having possible or probable SAMS, 65 of $76(86 \%)$ patients who did not test positive for SAMS were classified as unlikely with the index such that negative predictive value was $76.5 \%$. Lowering the cutoff for classification as unlikely to 4 from 6 points increased negative predictive value to $90.6 \%$. These preliminary results suggest that the SAMS-CI can be used to identify patients with selfreported SAMS who are unlikely to have true SAMS, perhaps improving the diagnosis of statin intolerance and encouraging statin adherence. The SAMS-CI needs to be validated prospectively in large clinical trials, as well as in clinical practice, however, before wide-scale adoption can be recommended.

Alternatively, the ACC statin intolerance tool, which is based on the 2013 ACC/AHA Guideline on the Treatment of Blood Cholesterol to Reduce Atherosclerotic Cardiovascular Risk in Adults [53], is available in both a web-based platform (http://tools.acc.org/statinintolerance/\#!/) as well as a mobile app. This tool seeks to categorize muscle symptoms in 8 different categories into a dichotomous outcome (possible vs unlikely) based on the following factors: symptom timing, symptom type, symptom location, sex, age, race/ethnicity, CK elevations $>5 \times$ upper limit of normal, known risk factors for SAMS, and non-statin causes of SAMS. After entering information in these 8 categories, the tool provides a summary indicating the number of factors classified as either possible or unlikely. Clinician and patient discretion is encouraged following the visual representation of the data to decide the likelihood of statin intolerance and the next potential treatment steps, such as dechallenge/rechallenge, reducing dose, or switching statins. The major drawback of the app is that it lacks a scoring system to differentiate the categorizations of possible versus unlikely into a diagnostic cutoff. Nonetheless, both the SAMS-CI and the ACC statin intolerance tool appear promising strategies that can be used to improve the distinction between true SAMS and nonspecific muscle pain or the nocebo effect.

\section{What Are the Most Effective Treatment Strategies for Managing SAMS and Encouraging Statin Adherence?}

The majority ( $\sim 60 \%$ ) of adults who discontinue statins reports SAMS as the primary reason for statin non-adherence and discontinuation [54]. The adverse impact of SAMS on optimal statin dosing and adherence has substantial health impacts. For example, among 45,037 adults with documented statin intolerance between 2006 and 2012, 25.5\% discontinued their statin prescription and $30.0 \%$ altered their statin therapy (with $52.6 \%$ down-titrating and $17.2 \%$ up-titrating their dose). Patients who discontinued their treatment exhibited the greatest LDL-C increases and were least likely to achieve an LDL-C $<100 \mathrm{mg} / \mathrm{dL}$ and $<70 \mathrm{mg} / \mathrm{dL}$ [55]. Moreover, statin intolerance is associated with a $36 \%$ higher rate of recurrent MI, a $43 \%$ higher rate of CHD events, and a $15 \%$ higher rate of all-cause mortality [14]. Therefore, clinicians must use a variety of strategies to manage and treat SAMS.

Managing the patient with possible SAMS requires a sequence of steps, which may vary by clinician and patient. Nonetheless, treatment requires reassessing the benefit of statin therapy, making the tentative diagnosis, eliminating contributing factors, reassuring the patient that SAMS are not permanent, trying alternative statins and doses, and prescribing alternative treatment strategies (Fig. 2) [56]. True SAMS is more likely when the typical clinical features are present [26, 51], so a careful documentation of symptoms is the initial diagnostic step. In addition, there is almost uniform agreement 


\section{Statin-Associated Muscle Symptom Clinical Index (SAMS-CI)}

\section{Instructions:}

- Use with patients who have had muscle symptoms that were new or increased after starting a statin regimen.

- A statin regimen includes any statin at any dose or frequency, including a statin the patient has used previously, at the same or a different dose.

- Muscle symptoms may include aches, cramps, heaviness, discomfort, weakness, or stiffness.

- Interpret overall score in light of other possible causes of the muscle symptoms, such as:
Recent physical exertion
Hypothyroidism
Concurrent illness
Changes in exercise patterns
Drug interaction with statin
Underlying muscle disease

- See reverse for Frequently Asked Questions

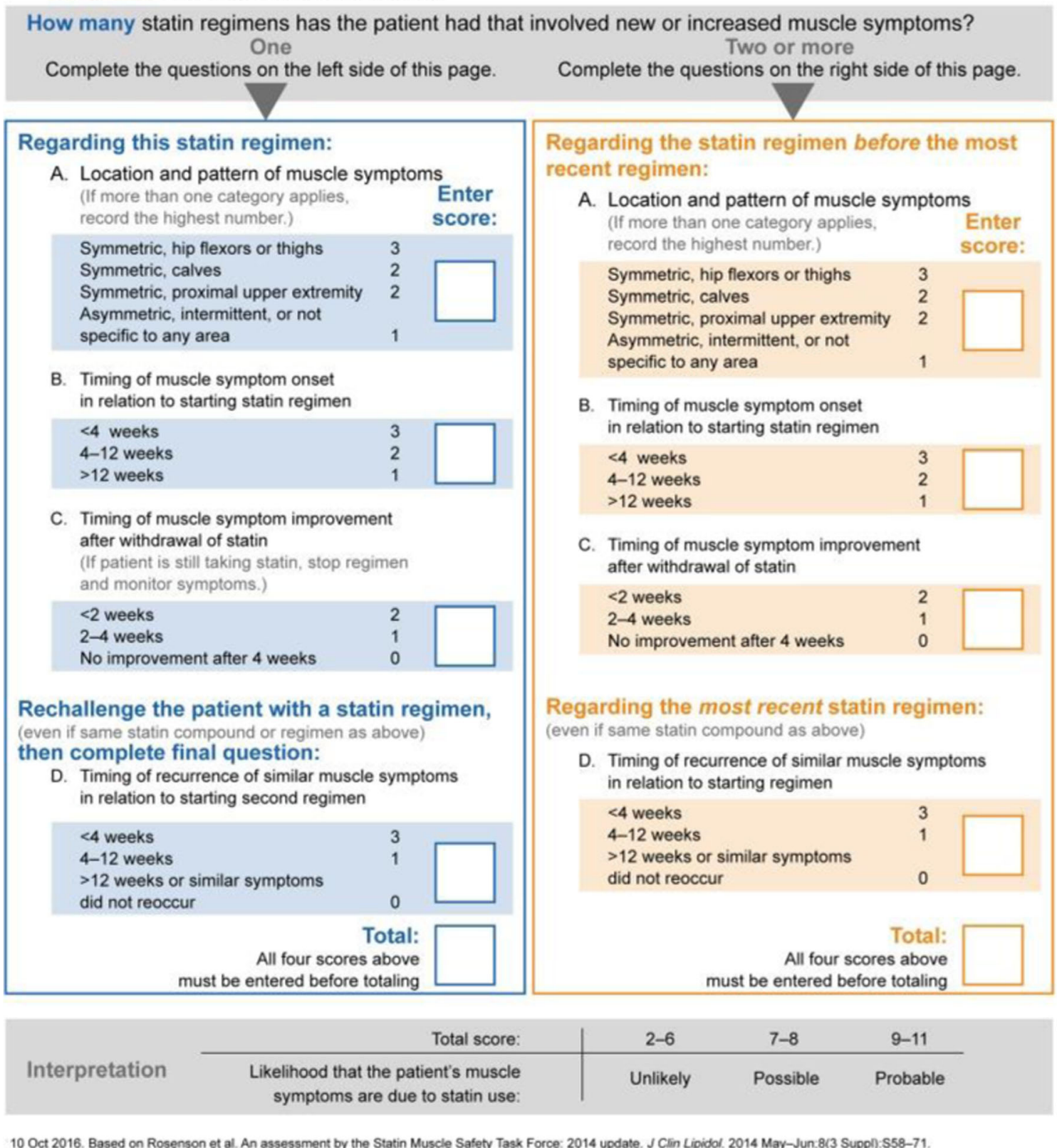

Fig. 1 The Statin-Associated Muscle Symptom Clinical Index (SAMSCI). The SAMS-CI [51] is distributed under the terms of the Creative Commons Attribution 4.0 International License (http://

that any definition of SAMS requires a dechallenge and rechallenge phase to assess potential causal associations, as well as attempting to use several $(\geq 2)$ different statins to support creativecommons.org/licenses/by/4.0/), which permits unrestricted use, distribution, and reproduction in any medium

the diagnosis [27]. The dechallenge phase should ideally involve statin cessation until symptoms have resolved (measuring the time course of symptom resolution), as well as some 
measurement of general muscle pain or status so that a true symptom "baseline" can be established prior to reinitiating a statin. This approach is critical because many patients have background chronic levels of muscle pain and symptoms associated with other conditions or medications. Repeated CK measurements can be beneficial to exclude clinically threatening muscle injury and to assist with the diagnosis because small increases in CK levels from baseline ( $20 \mathrm{U} / \mathrm{L})$ may help identify individuals who are truly experiencing SAMS, although again the large intra-individual variability in the measurement must be taken into consideration when interpreting changes with statin therapy [33]. It is equally important to exclude potentially contributing factors such as hypothyroidism and other medications and to evaluate the patient for other muscle diseases and conditions.

Careful determination of vitamin D status should also be included in the initial evaluation of SAMS, although the relationship between vitamin D deficiency and SAMS is equivocal. Vitamin D deficiency alone can cause skeletal myopathy [57] as well as decreased muscle strength [58, 59]. Consequently, vitamin D deficiency may exacerbate statin muscle symptoms and several studies and systematic reviews [60-64] have linked low vitamin D levels to SAMS. Vitamin D supplementation may mitigate muscle symptoms $[65,66]$, although this is not a consistent finding [67-69]. In our CoQ10 in Statin Myopathy Trial, we found no relationship between vitamin D levels, pre- and post-statin therapy, or clinical vitamin D deficiency and insufficiency, on the development of SAMS [70]. Nonetheless, vitamin D repletion when appropriate is recommended given the overall importance of vitamin D for muscle function.

Finally, an overlooked but critical step in the initial diagnosis and treatment of SAMS involves reassuring patients that statins are extremely safe and effective and that SAMS is reversible with drug cessation. Many patients are concerned about statin side effects, and negative media reports about statins are associated with their early discontinuation. For example, a Danish study investigated 674,900 individuals aged 40 or older who were initiated on statin therapy in 1995-2010 and followed them to 2011. Statin use increased from $<1$ to $11 \%$ and early statin discontinuation increased from 6 to $18 \%$ in the study population from 1995 to 2010. Odds ratios for early statin discontinuation versus continued use were 1.09 (95\% confidence interval, 1.06-1.12) in relation to the number of published negative statin-related news stories, demonstrating that increasing attention to potential statin adverse side effects is likely creating a nocebo effect [71]. This nocebo effect, the opposite of the placebo effect, almost certainly contributes to some patient's complaints during statin therapy [23]. Indeed, primary care physicians report that SAMS are the most common reason patients report for statin discontinuation, and patient fear of side effects is ranked by $70 \%$ of these physicians as the biggest challenge in persuading patients to reconsider statin therapy [72]. Promisingly, many patients can tolerate the drugs once the fear that the symptoms will progress and become permanent is addressed: over $90 \%$ of patients with statin complaints managed in an academic medical center were subsequently able to tolerate a statin [73].

After thorough diagnostic steps, 2 pharmaceutical strategies can be used to treat blood lipids to goal in the patient truly experiencing SAMS: alternate-day dosing and use of other, non-statin medications alone or in combination with lowdose statin therapy. Statins with longer half-lives such as rosuvastatin, atorvastatin, and pitavastatin can be given every other day or even less frequently [74]. A meta-analysis of 12 RCTs and 1 quasi-RCT ( $n=1023$ patients) revealed no statistically significant difference between alternate-day and daily regimens of atorvastatin and rosuvastatin in terms of change in LDL-C and triglycerides, although daily regimens of atorvastatin and rosuvastatin were superior to alternate-day regimes for change in total cholesterol. In addition, there was no statistically significant difference between alternate-day and daily regimens on all lipid outcomes for both fluvastatin and pravastatin [75]. It should be noted that the majority of trials included in this meta-analysis used the same dose of statin (i.e., $10 \mathrm{mg}$ both daily and on alternate days) so that alternate-day dosing resulted in a lower total weekly dose. Consequently, clinicians should consider alternate-day dosing a cornerstone for managing elevated cholesterol and reducing cardiovascular risk in patients with SAMS.

Among the most commonly used non-statin medications are ezetimibe and monoclonal antibody proprotein convertase subtilisin/kexin type 9 (PCSK9) inhibitors. The 2016 American College of Cardiology Expert Consensus regarding the use of non-statin therapies for LDL-C lowering suggests the addition of ezetimibe as the first non-statin agent for patients not at lipid goals [76], and most studies have shown that ezetimibe does not increase SAMS in statin-intolerant patients $[22,77]$. The 2 approved PCSK 9 inhibitors (alirocumab and evolocumab) are used for lowering LDL-C in patients who are unable to reach desired LDL-C levels with diet and maximally tolerated lipid-lowering therapy alone. These PCSK9 inhibitors reduce LDL-C by an additional 50 to $70 \%$ in patients on statin (and other lipid-lowering) therapy, and several landmark trials involving statin-intolerant patients have demonstrated that the rates of SAMS and resultant drug discontinuation are equal to and perhaps less than rates observed with statin therapy alone $[22,77,78]$. For example, in the GAUSS-3 trial, which enrolled patients with a history of statin intolerance to $\geq 2$ statins, atorvastatin treatment evoked muscle symptoms in 209 of 491 (42.6\%) of patients on atorvastatin but not placebo in the first phase of the study. These patients were then randomized to ezetimibe versus evolocumab, where muscle symptoms were reported in $28.8 \%$ of ezetimibe-treated patients and $20.7 \%$ of evolocumab-treated patients [22]. In the Odyssey Alternative trial, SAMS occurred less frequently 

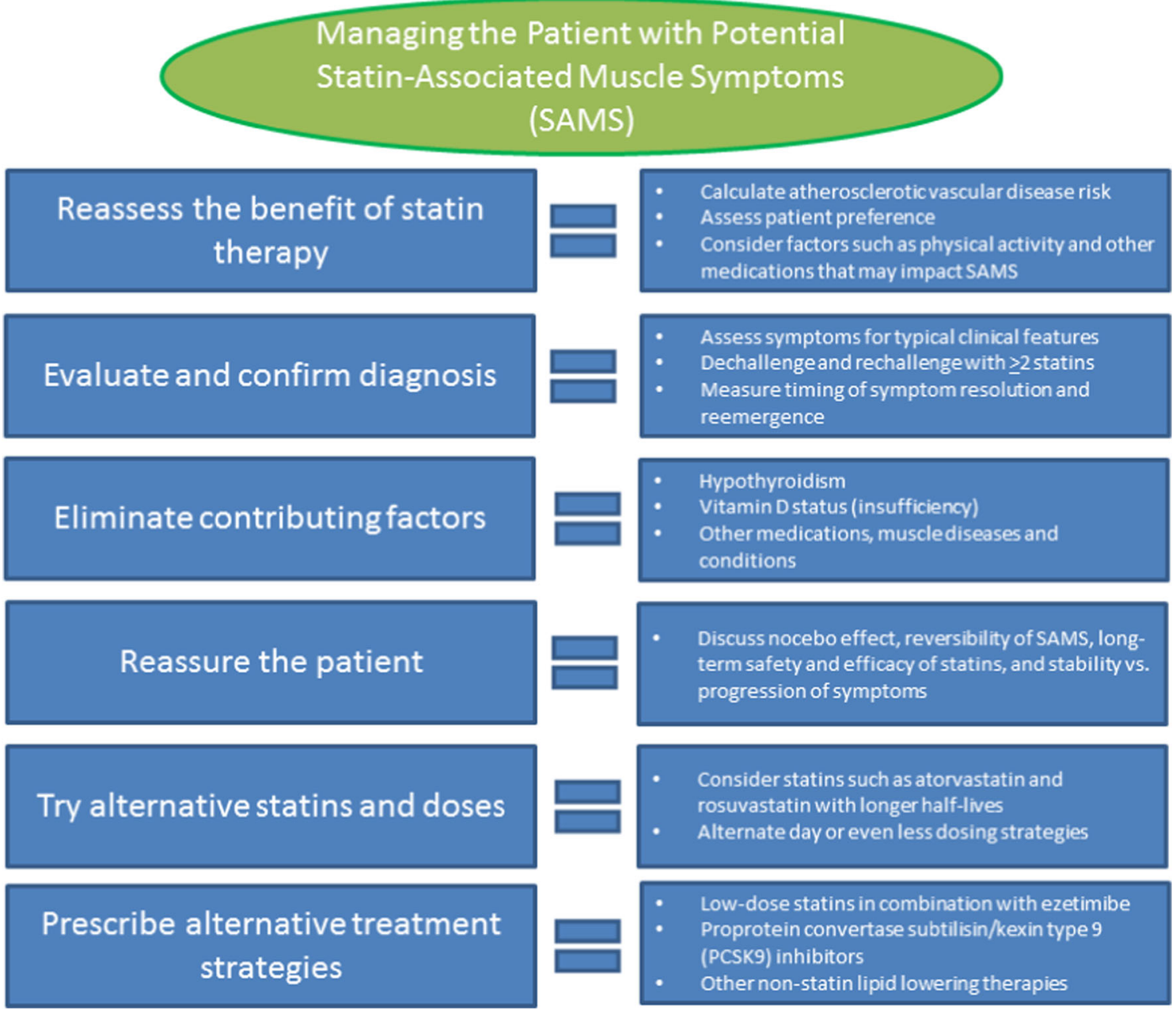

Fig. 2 A schematic of the diagnosis, treatment, and management strategies for SAMS

with alirocumab (32.5\%) versus atorvastatin $(46.0 \%)$, although treatment discontinuation due to SAMS was not different between the 2 treatments [77]. The recent FDA approval of evolocumab for expanded use to prevent heart attacks, strokes, and coronary revascularizations in adults with established cardiovascular disease [79] indicates that PCSK9 inhibitors will continue to gain widespread use, particularly in patients with SAMS. Finally, alternative lipid-lowering regimens including niacin, cholestyramine, fenofibrate, and gemfibrozil, which are less favored due to ambiguous early trial results, potential interactions with statins, and other adverse side effects, can be considered when statins are not tolerated as more recent evidence and review of the data indicates they should not be wholly excluded as treatment options [56].

There is a paucity of data addressing the efficacy of nutraceutical supplementation for patients with SAMS [80]. The most thoroughly investigated is CoQ10 supplementation, which is one of the most widely popular treatments for reducing SAMS. Multiple trials have evaluated the impact of coenzyme Q10 supplementation on statin-associated muscle symptoms with approximately half showing a benefit and the other half showing no effect [21, 81-89]. When we placed subjects with confirmed SAMS on either simvastatin $20 \mathrm{mg}$ daily plus CoQ10 or placebo for 8 weeks [21], CoQ10 supplementation had no effect on the incidence and severity of SAMS, time to onset of pain, muscle strength, or aerobic performance. Similarly, a recent meta-analysis of $5 \mathrm{CoQ} 10$ supplementation trials with 253 participants found no significant effect of CoQ10 on SAMS despite a small, non-significant trend $(p=$ 0.20 ) toward a decrease in muscle pain [90]. A study investigating the effect of CoQ10 supplementation on phosphocreatine $(\mathrm{PCr}$ ) recovery kinetics (a more direct assessment of muscle mitochondrial function) found no impact of $\mathrm{CoQ} 10 \mathrm{on} \mathrm{PCr}$ kinetics compared to statin therapy alone [88]. Collectively, data do not strongly support the concept that CoQ10 supplementation mitigates SAMS.

\section{Future Directions and Conclusions}

This review summarized recent evidence related to diagnosis and management of SAMS. Several major themes emerge 
with respect to gaps in knowledge and future directions. Given the uncertainties surrounding the true prevalence of SAMS, and the almost certain fact that many reports and studies of patients with SAMS have enrolled patients with non-specific muscle complaints and/or the nocebo effect, future trials need to more accurately assess SAMS in study participants via a rigorous dechallenge-rechallenge protocol or double-blind, placebo-controlled statin run-in study. This will improve reporting of SAMS rates and also ensure that trials of statin intolerance enroll only true patients with SAMS. Moreover, a biomarker of SAMS is desperately needed in order to aid clinicians in the diagnosis of SAMS and to improve statin adherence in patients not truly statin intolerant. Small changes in study design, such as uniformly assessing and reporting CK levels before and after statin treatment, could greatly increase the likelihood of such a biomarker being found. And finally, long-term outcome trials of various statin and non-statin regimens in patients with SAMS are still lacking. These large outcomes trials are necessary to develop a consensus treatment plan that weighs the costs and benefits of statin use versus other pharmacological and non-pharmacological options for the long-term reduction of atherosclerotic cardiovascular disease events.

Required Author Forms Disclosure forms provided by the authors are available with the online version of this article.

\section{Compliance with Ethical Standards}

Disclosures Dr. Thompson has received research support from Sanofi, Regeneron, Esperion, and Amarin; has served as a consultant for Amgen, Regeneron, Sanofi, Esperion, and Amarin; has received speaker honoraria from Kowa, Amarin, Amgen, Regeneron, and Sanofi; owns stock in Abbvie, Abbott Labs, Boston Scientific, General Electric, J\&J, Myokardia, and Serapta; and has provided expert legal testimony on exercise-related cardiac events and statin myopathy. Dr. Taylor served on the Pharmacovigilance Monitoring Board for Amgen, Inc., and received grant support from Regeneron, Inc.

\section{References}

1. National Center for Health Statistics. 2015; Available at: www.cdc. gov/nhs. Accessed 11/20, 2015.

2. Randomised trial of cholesterol lowering in 4444 patients with coronary heart disease: the Scandinavian Simvastatin Survival Study (4S). Lancet. 1994;344:1383-1389.

3. Downs JR, Clearfield M, Weis S, Whitney E, Shapiro DR, Beere PA, et al. Primary prevention of acute coronary events with lovastatin in men and women with average cholesterol levels: results of AFCAPS/TexCAPS. Air Force/Texas Coronary Atherosclerosis Prevention Study. JAMA 1998;279:1615-1622.

4. Pencina MJ, Navar-Boggan AM, D'Agostino RB S, Williams K, Neely B, Sniderman AD, et al. Application of new cholesterol guidelines to a population-based sample. N Engl J Med 2014;370: 1422-1431.
5. Ueda P, Lung TW, Lu Y, Salomon JA, Rahimi K, Clarke P, et al. Treatment gaps and potential cardiovascular risk reduction from expanded statin use in the US and England. PLoS One 2018;13: e0190688.

6. Cziraky MJ, Willey VJ, McKenney JM, Kamat SA, Fisher MD, Guyton JR, et al. Risk of hospitalized rhabdomyolysis associated with lipid-lowering drugs in a real-world clinical setting. J Clin Lipidol 2013;7:102-108.

7. Cziraky MJ, Willey VJ, McKenney JM, Kamat SA, Fisher MD, Guyton JR, et al. Statin safety: an assessment using an administrative claims database. Am J Cardiol 2006;97:61C-68C.

8. Mohassel P, Mammen AL. Anti-HMGCR Myopathy. J Neuromuscul Dis 2018;5:11-20.

9. Grable-Esposito P, Katzberg HD, Greenberg SA, Srinivasan J, Katz $\mathrm{J}$, Amato AA. Immune-mediated necrotizing myopathy associated with statins. Muscle Nerve 2010;41:185-190.

10. Mammen AL, Chung T, Christopher-Stine L, Rosen P, Rosen A, Doering KR, et al. Autoantibodies against 3-hydroxy-3methylglutaryl-coenzyme A reductase in patients with statinassociated autoimmune myopathy. Arthritis Rheum 2011;63:713721.

11. Musset L, Allenbach Y, Benveniste O, Boyer O, Bossuyt X, Bentow $\mathrm{C}$, et al. Anti-HMGCR antibodies as a biomarker for immune-mediated necrotizing myopathies: A history of statins and experience from a large international multi-center study. Autoimmun Rev 2016;15:983-993.

12. Cohen JD, Brinton EA, Ito MK, Jacobson TA. Understanding Statin Use in America and Gaps in Patient Education (USAGE): an internet-based survey of 10,138 current and former statin users. J Clin Lipidol 2012;6:208-215.

13. Franklin JM, Krumme AA, Tong AY, Shrank WH, Matlin OS, Brennan TA, et al. Association between trajectories of statin adherence and subsequent cardiovascular events. Pharmacoepidemiol Drug Saf 2015;24:1105-1113.

14. Serban MC, Colantonio LD, Manthripragada AD, Monda KL, Bittner VA, Banach M, et al. Statin Intolerance and Risk of Coronary Heart Events and All-Cause Mortality Following Myocardial Infarction. J Am Coll Cardiol 2017;69:1386-1395.

15. Graham JH, Sanchez RJ, Saseen JJ, Mallya UG, Panaccio MP, Evans MA. Clinical and economic consequences of statin intolerance in the United States: Results from an integrated health system. J Clin Lipidol 2017;11:70-79.e1.

16. Pittman DG, Chen W, Bowlin SJ, Foody JM. Adherence to statins, subsequent healthcare costs, and cardiovascular hospitalizations. Am J Cardiol 2011;107:1662-1666.

17. Bruckert E, Hayem G, Dejager S, Yau C, Begaud B. Mild to moderate muscular symptoms with high-dosage statin therapy in hyperlipidemic patients-the PRIMO study. Cardiovasc Drugs Ther 2005; 19:403-414.

18. Buettner C, Davis RB, Leveille SG, Mittleman MA, Mukamal KJ. Prevalence of musculoskeletal pain and statin use. J Gen Intern Med 2008;23:1182-1186.

19. Ganga HV, Slim HB, Thompson PD. A systematic review of statininduced muscle problems in clinical trials. Am Heart J 2014;168:615.

20. Parker BA, Capizzi JA, Grimaldi AS, Clarkson PM, Cole SM, Keadle J, et al. Effect of statins on skeletal muscle function. Circulation 2013;127:96-103.

21. Taylor BA, Lorson L, White CM, Thompson PD. A randomized trial of coenzyme Q10 in patients with confirmed statin myopathy. Atherosclerosis 2015;238:329-335.

22. Nissen SE, Stroes E, Dent-Acosta RE, Rosenson RS, Lehman SJ, Sattar N, et al. Efficacy and Tolerability of Evolocumab vs Ezetimibe in Patients With Muscle-Related Statin Intolerance: The GAUSS-3 Randomized Clinical Trial. JAMA 2016;315: $1580-1590$. 
23. Tobert JA, Newman CB. The nocebo effect in the context of statin intolerance. J Clin Lipidol 2016;10:739-747.

24. Slomski A. Nocebo Effect May Account for Statin Adverse Events. JAMA 2017;317:2476.

25. Herrett E, Williamson E, Beaumont D, Prowse D, Youssouf N, Brack K, et al. Study protocol for statin web-based investigation of side effects (StatinWISE): a series of randomised controlled Nof-1 trials comparing atorvastatin and placebo in UK primary care. BMJ Open 2017;7:e16604-2017-016604.

26. Rosenson RS, Baker SK, Jacobson TA, Kopecky SL, Parker BA, The National Lipid Association's Muscle Safety Expert,Panel. An assessment by the Statin Muscle Safety Task Force: 2014 update. J Clin Lipidol 2014;8:S58-S71.

27. Rosenson RS, Baker S, Banach M, Borow KM, Braun LT, Bruckert E, et al. Optimizing Cholesterol Treatment in Patients With Muscle Complaints. J Am Coll Cardiol 2017;70:1290-1301.

28. Cho L, Rocco M, Colquhoun D, Sullivan D, Rosenson RS, Dent R, et al. Clinical Profile of Statin Intolerance in the Phase 3 GAUSS-2 Study. Cardiovasc Drugs Ther 2016;30:297-304.

29. Cham S, Evans MA, Denenberg JO, Golomb BA. Statin-associated muscle-related adverse effects: a case series of 354 patients. Pharmacotherapy 2010;30:541-553.

30. Tiwari A, Bansal V, Chugh A, Mookhtiar K. Statins and myotoxicity: a therapeutic limitation. Expert Opin Drug Saf 2006;5:651-666.

31. Mancini GB, Baker S, Bergeron J, Fitchett D, Frohlich J, Genest J, et al. Diagnosis, Prevention, and Management of Statin Adverse Effects and Intolerance: Canadian Consensus Working Group Update (2016). Can J Cardiol 2016;32:S35-S65.

32. Ballard KD, Parker BA, Capizzi JA, Grimaldi AS, Clarkson PM, Cole SM, et al. Increases in creatine kinase with atorvastatin treatment are not associated with decreases in muscular performance. Atherosclerosis 2013;230:121-124.

33. Taylor BA, Panza G, Thompson PD. Increased creatine kinase with statin treatment may identify statin-associated muscle symptoms. Int J Cardiol 2016;209:12-13.

34. Sinzinger H, O'Grady J. Professional athletes suffering from familial hypercholesterolaemia rarely tolerate statin treatment because of muscular problems. Br J Clin Pharmacol 2004;57:525-528.

35. Thompson PD, Gadaleta PA, Yurgalevitch S, Cullinane E, Herbert PN. Effects of exercise and lovastatin on serum creatine kinase activity. Metabolism 1991;40:1333-1336.

36. Kearns AK, Bilbie CL, Clarkson PM, White CM, Sewright KA, O'Fallon KS, et al. The creatine kinase response to eccentric exercise with atorvastatin $10 \mathrm{mg}$ or $80 \mathrm{mg}$. Atherosclerosis 2008;200: 121-125.

37. Parker BA, Augeri AL, Capizzi JA, Ballard KD, Troyanos C, Baggish AL, et al. Effect of statins on creatine kinase levels before and after a marathon run. Am J Cardiol 2012;109:282-287.

38. Thompson PD, Zmuda JM, Domalik LJ, Zimet RJ, Staggers J, Guyton JR. Lovastatin increases exercise-induced skeletal muscle injury. Metabolism 1997:46:1206-1210.

39. Krishnan GM, Thompson PD. The effects of statins on skeletal muscle strength and exercise performance. Curr Opin Lipidol 2010;21:324-328.

40. Scott D, Blizzard L, Fell J, Jones G. Statin therapy, muscle function and falls risk in community-dwelling older adults. QJM 2009;102: 625-633.

41. Panza GA, Taylor BA, Dada MR, Thompson PD. Changes in muscle strength in individuals with statin-induced myopathy: A summary of 3 investigations. J Clin Lipidol 2015;9:351-356.

42. Taylor BA, Thompson PD. Muscle-related side-effects of statins: from mechanisms to evidence-based solutions. Curr Opin Lipidol 2015;26:221-227.

43. Mallinson JE, Marimuthu K, Murton A, Selby A, Smith K, Constantin-Teodosiu D, et al. Statin myalgia is not associated with reduced muscle strength, mass or protein turnover in older male volunteers, but is allied with a slowing of time to peak power output, insulin resistance and differential muscle mRNA expression. J Physiol 2015;593:1239-1257.

44. Bouitbir J, Charles AL, Rasseneur L, Dufour S, Piquard F, Geny B, et al. Atorvastatin treatment reduces exercise capacities in rats: involvement of mitochondrial impairments and oxidative stress. J Appl Physiol (1985) 2011;111:1477-1483.

45. Bouitbir J, Singh F, Charles AL, Schlagowski AI, Bonifacio A, Echaniz-Laguna A, et al. Statins Trigger Mitochondrial Reactive Oxygen Species-Induced Apoptosis in Glycolytic Skeletal Muscle. Antioxid Redox Signal 2016;24:84-98.

46. Hubal MJ, Reich KA, De Biase A, Bilbie C, Clarkson PM, Hoffman EP, et al. Transcriptional deficits in oxidative phosphorylation with statin myopathy. Muscle Nerve 2011;44:393-401.

47. Wu JS, Buettner C, Smithline H, Ngo LH, Greenman RL. Evaluation of skeletal muscle during calf exercise by 31 phosphorus magnetic resonance spectroscopy in patients on statin medications. Muscle Nerve 2011;43:76-81.

48. Grassi B, Porcelli S, Marzorati M, Lanfranconi F, Vago P, Marconi $\mathrm{C}$, et al. Metabolic myopathies: functional evaluation by analysis of oxygen uptake kinetics. Med Sci Sports Exerc 2009;41:2120-2127.

49. Mikus CR, Boyle LJ, Borengasser SJ, Oberlin DJ, Naples SP, Fletcher J, et al. Simvastatin impairs exercise training adaptations. J Am Coll Cardiol 2013;62:709-714.

50. Ballard KD, Mazur SM, Taylor BA, Thompson PD. Short-term cessation of statin therapy does not alter aerobic exercise performance in physically active middle-aged adults. Phys Sportsmed 2016;44:63-67.

51. Rosenson RS, Miller K, Bayliss M, Sanchez RJ, Baccara-Dinet MT, Chibedi-De-Roche D, et al. The Statin-Associated Muscle Symptom Clinical Index (SAMS-CI): Revision for Clinical Use, Content Validation, and Inter-rater Reliability. Cardiovasc Drugs Ther 2017;31:179-186.

52. Taylor BA, Sanchez RJ, Jacobson TA, Chibedi-De-Roche D, Manvelian G, Baccara-Dinet MT, et al. Application of the StatinAssociated Muscle Symptoms-Clinical Index to a Randomized Trial onStatin Myopathy. J Am Coll Cardiol 2017;70:1680-1681.

53. Stone NJ, Robinson JG, Lichtenstein AH, Bairey Merz CN, Blum $\mathrm{CB}$, Eckel RH, et al. 2013 ACC/AHA guideline on the treatment of blood cholesterol to reduce atherosclerotic cardiovascular risk in adults: a report of the American College of Cardiology/American Heart Association Task Force on Practice Guidelines. Circulation 2014;129:S1-S45.

54. Wei MY, Ito MK, Cohen JD, Brinton EA, Jacobson TA. Predictors of statin adherence, switching, and discontinuation in the USAGE survey: understanding the use of statins in America and gaps in patient education. J Clin Lipidol 2013;7:472-483.

55. Harrison TN, Hsu JY, Rosenson RS, Levitan EB, Muntner P, Cheetham TC, et al. Unmet Patient Need in Statin Intolerance: the Clinical Characteristics and Management. Cardiovasc Drugs Ther 2018;32:29-36.

56. Thompson PD, Panza G, Zaleski A, Taylor B. Statin-Associated Side Effects. J Am Coll Cardiol 2016;67:2395-2410.

57. Ziambaras K, Dagogo-Jack S. Reversible muscle weakness in patients with vitamin D deficiency. West J Med 1997;167:435-439.

58. Mastaglia SR, Seijo M, Muzio D, Somoza J, Nunez M, Oliveri B. Effect of vitamin D nutritional status on muscle function and strength in healthy women aged over sixty-five years. J Nutr Health Aging 2011;15:349-354.

59. Marantes I, Achenbach SJ, Atkinson EJ, Khosla S, Melton LJ, 3rd, Amin S. Is vitamin D a determinant of muscle mass and strength? J Bone Miner Res 2011;26:2860-2871.

60. Michalska-Kasiczak M, Sahebkar A, Mikhailidis DP, Rysz J, Muntner P, Toth PP, et al. Analysis of vitamin D levels in patients with and without statin-associated myalgia - a systematic review 
and meta-analysis of 7 studies with 2420 patients. Int J Cardiol 2015;178:111-116.

61. Pereda CA, Nishishinya MB. Is there really a relationship between serum vitamin D (25OHD) levels and the musculoskeletal pain associated with statin intake? A systematic review. Reumatol Clin. 2016;12:331-335.

62. Mergenhagen K, Ott M, Heckman K, Rubin LM, Kellick K. Low vitamin $\mathrm{D}$ as a risk factor for the development of myalgia in patients taking high-dose simvastatin: a retrospective review. Clin Ther 2014;36:770-777.

63. Ovesjo ML, Skilving I, Bergman P, Rane A, Ekstrom L, BjorkhemBergman L. Low Vitamin D Levels and Genetic Polymorphism in the Vitamin D Receptor are Associated with Increased Risk of Statin-Induced Myopathy. Basic Clin Pharmacol Toxicol 2016;118:214-218.

64. Morioka TY, Lee AJ, Bertisch S, Buettner C. Vitamin D status modifies the association between statin use and musculoskeletal pain: a population based study. Atherosclerosis 2015;238:77-82.

65. Khayznikov M, Hemachrandra K, Pandit R, Kumar A, Wang P, Glueck CJ. Statin Intolerance Because of Myalgia, Myositis, Myopathy, or Myonecrosis Can in Most Cases be Safely Resolved by Vitamin D Supplementation. N Am J Med Sci 2015;7:86-93.

66. Jetty V, Glueck CJ, Wang P, Shah P, Prince M, Lee K, et al. Safety of 50,000-100,000 Units of Vitamin D3/Week in Vitamin DDeficient, Hypercholesterolemic Patients with Reversible Statin Intolerance. N Am J Med Sci 2016;8:156-162.

67. Kurnik D, Hochman I, Vesterman-Landes J, Kenig T, Katzir I, Lomnicky Y, et al. Muscle pain and serum creatine kinase are not associated with low serum $25(\mathrm{OH})$ vitamin D levels in patients receiving statins. Clin Endocrinol (Oxf) 2012;77:36-41.

68. Eisen A, Lev E, Iakobishvilli Z, Porter A, Brosh D, Hasdai D, et al. Low plasma vitamin D levels and muscle-related adverse effects in statin users. Isr Med Assoc J 2014;16:42-45.

69. Riphagen IJ, van der Veer E, Muskiet FA, DeJongste MJ. Myopathy during statin therapy in the daily practice of an outpatient cardiology clinic: prevalence, predictors and relation with vitamin D. Curr Med Res Opin 2012;28:1247-1252.

70. Taylor B, Lorson L, White M, Thompson PD. Low vitamin D does not predict statin-associated muscle symptoms but is associated with transient increases in muscle damage and pain. Atherosclerosis 2016; 256:100-104.

71. Nielsen SF, Nordestgaard BG. Negative statin-related news stories decrease statin persistence and increase myocardial infarction and cardiovascular mortality: a nationwide prospective cohort study. Eur Heart J 2016;37:908-916.

72. Tanner RM, Safford MM, Monda KL, Taylor B, O'Beirne R, Morris $\mathrm{M}$, et al. Primary Care Physician Perspectives on Barriers to Statin Treatment. Cardiovasc Drugs Ther 2017;31:303-309.

73. Zhang H, Plutzky J, Shubina M, Turchin A. Continued Statin Prescriptions After Adverse Reactions and Patient Outcomes: A Cohort Study. Ann Intern Med 2017;167:221-227.

74. Juszczyk MA, Seip RL, Thompson PD. Decreasing LDL cholesterol and medication cost with every-other-day statin therapy. Prev Cardiol 2005;8:197-199.

75. Awad K, Mikhailidis DP, Toth PP, Jones SR, Moriarty P, Lip GYH, et al. Efficacy and Safety of Alternate-Day Versus Daily Dosing of Statins: a Systematic Review and Meta-Analysis. Cardiovasc Drugs Ther 2017;31:419-431.
76. Writing Committee, Lloyd-Jones DM, Morris PB, Ballantyne CM, Birtcher KK, Daly DD, Jr, et al. 2016 ACC Expert Consensus Decision Pathway on the Role of Non-Statin Therapies for LDLCholesterol Lowering in the Management of Atherosclerotic Cardiovascular Disease Risk: A Report of the American College of Cardiology Task Force on Clinical Expert Consensus Documents. J Am Coll Cardiol 2016;68:92-125.

77. Moriarty PM, Thompson PD, Cannon CP, Guyton JR, Bergeron J, Zieve FJ, et al. Efficacy and safety of alirocumab vs ezetimibe in statin-intolerant patients, with a statin rechallenge arm: The ODYSSEY ALTERNATIVE randomized trial. J Clin Lipidol 2015:9:758-769.

78. Stroes E, Colquhoun D, Sullivan D, Civeira F, Rosenson RS, Watts GF, et al. Anti-PCSK9 antibody effectively lowers cholesterol in patients with statin intolerance: the GAUSS-2 randomized, placebo-controlled phase 3 clinical trial of evolocumab. J Am Coll Cardiol 2014;63:2541-2548.

79. Sabatine MS, Giugliano RP, Keech AC, Honarpour N, Wiviott SD, Murphy SA, et al. Evolocumab and Clinical Outcomes in Patients with Cardiovascular Disease. N Engl J Med 2017;376:1713-1722.

80. Banach M, Patti AM, Giglio RV, Cicero AFG, Atanasov AG, Bajraktari G, et al. The Role of Nutraceuticals in Statin Intolerant Patients. J Am Coll Cardiol 2018;72:96-118.

81. Caso G, Kelly P, McNurlan MA, Lawson WE. Effect of coenzyme q10 on myopathic symptoms in patients treated with statins. Am J Cardiol 2007;99:1409-1412.

82. Young JM, Florkowski CM, Molyneux SL, McEwan RG, Frampton CM, George PM, et al. Effect of coenzyme Q(10) supplementation on simvastatin-induced myalgia. Am J Cardiol 2007;100:1400-1403.

83. Bookstaver DA, Burkhalter NA, Hatzigeorgiou C. Effect of coenzyme Q10 supplementation on statin-induced myalgias. Am J Cardiol 2012;110:526-529.

84. Bogsrud MP, Langslet G, Ose L, Arnesen KE, Sm Stuen MC, Malt UF, et al. No effect of combined coenzyme Q10 and selenium supplementation on atorvastatin-induced myopathy. Scand Cardiovasc J 2013;47:80-87.

85. Fedacko J, Pella D, Fedackova P, Hanninen O, Tuomainen P, Jarcuska $P$, et al. Coenzyme $Q(10)$ and selenium in statinassociated myopathy treatment. Can J Physiol Pharmacol 2013;91:165-170.

86. Deichmann RE, Lavie CJ, Dornelles AC. Impact of coenzyme Q-10 on parameters of cardiorespiratory fitness and muscle performance in older athletes taking statins. Phys Sportsmed 2012;40:88-95.

87. Skarlovnik A, Janic M, Lunder M, Turk M, Sabovic M. Coenzyme Q10 supplementation decreases statin-related mild-to-moderate muscle symptoms: a randomized clinical study. Med Sci Monit 2014;20:2183-2188.

88. Buettner C, Greenman RL, Ngo LH, Wu JS. Effects of Coenzyme Q10 on Skeletal Muscle Oxidative Metabolism in Statin Users Assessed Using 31P Magnetic Resonance Spectroscopy: a Randomized Controlled Study. J Nat Sci 2016;2:e212.

89. Toth S, Sajty M, Pekarova T, Mughees A, Stefanic P, Katz M, et al. Addition of omega-3 fatty acid and coenzyme Q10 to statin therapy in patients with combined dyslipidemia. J Basic Clin Physiol Pharmacol 2017;28:327-336.

90. Banach M, Serban C, Sahebkar A, Ursoniu S, Rysz J, Muntner P, et al. Effects of coenzyme Q10 on statin-induced myopathy: a metaanalysis of randomized controlled trials: Mayo Clin Proc 2015;90: 24-34. 